\title{
A CONTINUOUS STUDY OF MORPHOLOGICAL PHASE IN THE SWARM OF PROTEUS
}

\author{
K. A. Bisset and C. W. I. Douglas \\ Department of Bacteriology, University of Birmingham, Birmingham B15 2TJ
}

\section{Plates XVII and XVIII}

THE classical explanation of the swarm-zonation phenomenon in Proteus is that the elongated, hyper-flagellate elements alternate at the edge of the swarm with short, non-swarming bacilli, and that this alternation leads to the formation of zones as the edge of the swarm periodically advances, halts and again advances. This general explanation was quoted as recently as 1974 by Armitage, Rowbury and Smith.

In a recent study of swarming and zonation in Proteus mirabilis (Bisset, 1973a and $b$ ), it was pointed out that the use of the phase-contrast microscope to examine the edge of the swarm, in situ and undisturbed upon the culture medium, led to a different conclusion. The bacteria at the edge of the swarm colony were found to be persistently in the swarm phase, irrespective of whether the swarm was or was not advancing, whereas the short, nonswarming phase appeared behind the edge where motility had ceased by reason of growth and overcrowding.

Because this observation contradicts a widely held belief, we produce experimental evidence in its support.

\section{MATERIALS AND METHODS}

Initial observations were made on approximately 10 strains of Proteus mirabilis and $P$. vulgaris in the course of earlier investigations (Bisset, 1973a and $b$; Wolstenholme, 1973). The present study was performed on six strains of $P$. vulgaris, freshly isolated in this laboratory and in the bacteriology laboratory of the Queen Elizabeth Hospital. Cultures were made by inoculating the centre of partially dried agar plates (BBL Trypticase Soy). These were enclosed individually in plastic bags and incubated at $37^{\circ} \mathrm{C}$ in a large warm cupboard, so that the operator could enter to manipulate the cultures without alteration of temperature.

At intervals of $1 \mathrm{~h}$, small pieces of agar $(c .2 \times 2 \mathrm{~mm})$ were removed with the point of a sterile knife, always from the position occupied by the extreme edge of the swarm at that instant; these were transferred face upwards to a slide, placed on the surface of a block of ice for 2-3 min. to inhibit motility, and photographed with the $\times 40$ lens of a phasecontrast microscope. A permanent record was obtained by inverting the piece of agar to make a smear which was dried and stained.

When the swarm had ceased to advance actively, after c. $12 \mathrm{~h}$., the culture was killed and preserved with formaldehyde vapour after comparative smears had been made from the growth adjacent to each space from which the agar had been removed, to give a record of the change in morphology since the original samples were taken.

Care was taken to remove the samples from areas distributed evenly around the swarm, while avoiding removing later tests from the track of portions of the swarm that might have been disturbed by previous sampling.

Received 14 Mar. 1975; accepted 6 Oct. 1975.

J. MED. MICROBIOL.-VOL. 9 (1976) 


\section{RESULTS}

Examples of swarms on agar plates, tested in this manner, are given in figs. 1 and 2, and it can be seen that the samples had been removed from areas which, although constituting a part of the edge of the swarm at the time when the test was made, represent all stages in the zonation pattern of the mature plate.

The inoculum, and the growth immediately derived from it before the beginning of swarming, consisted of short forms; thereafter, as seen by phase-contrast, in almost every case, the edge of the swarm consisted of filaments in a coiled " Medusa-head " arrangement, irrespective of whether the swarm could be observed to be in motion or not (figs. 3-5). In occasional samples (less than one per plate, usually) the swarm was completely at rest and in a loose formation; the edge nevertheless consisted almost entirely of elongated, swarm-phase elements (fig. 6). In this figure it can also be seen that the short, non-swarming elements appear at a distance behind the edge, and in an ordinary smear taken from such a specimen a mixture of long and shorter forms appears (fig. 7). The smears made from the sample areas after the completion of swarming consisted entirely of very short elements (fig. 8) in the interior parts of the colony, but with progressively more swarming elements towards the outer edge.

\section{Discussion}

It appears from the above evidence that the discrepancy between our own observations and the classical explanation of the swarm phenomenon in Proteus must lie in the fact that the edge of the swarm, consisting, in our experience, of swarm-type elements at all stages throughout the development of the swarm colony, may be very narrow. The relatively crude method traditionally employed, of taking a portion of growth with an inoculating loop and making a smear upon a slide, not only provides a sample at varying distances behind the edge but is liable so to disturb and mix the sample that the natural relationship of its various elements is obscured.

It is possible-indeed probable-that different strains of Proteus may behave differently, but the classical and universally accepted explanation of the zonation phenomenon is not strictly accurate. The present evidence tends to confirm the explanation offered by one of us (Bisset, 1973b), that the non-swarming phase is the result rather than the cause of cessation of motion at the edge of the swarm, that the actual edge consists of elongated swarmers, in motion or otherwise. A ring in the swarm pattern arises when non-swarming growth piles up behind the edge, and the swarm advances, leaving it behind. The discrepancy between these results and the classical explanation is probably attributable to the relative inaccuracy of sampling methods previously employed.

\section{SUMMARY}

Continuous study, at intervals of $1 \mathrm{~h}$, on the advancing edge of the swarm of Proteus vulgaris confirms that this is almost permanently composed of elongated swarmers, and that short, non-swarming forms arise in the interior of the culture where motion has already ceased. Previous errors have probably arisen from inaccurate sampling.

\section{REFERENCES}

Armitage, Judith P., Rowbury, R. J. and Smith, D. C. 1974 . The effects of chloramphenicol, nalidixic acid and penicillin on the growth and division of swarming cells of Proteus mirabilis. J. med. Microbiol., 7, 459.

BisseT, K. A. 1973a. The motion of the swarm in Proteus mirabilis. J. med. Microbiol., 6, 33 . 
SWARMing of Proteus
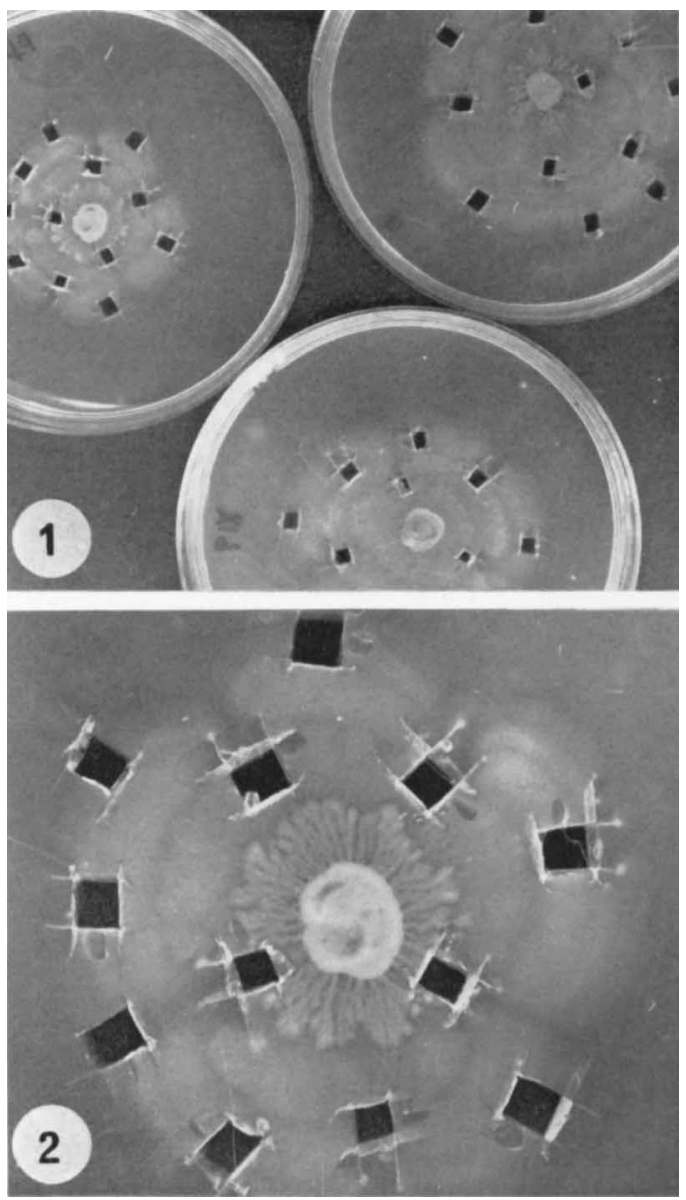

FiG. 1.-Examples of plates bearing swarms from which samples of the growth edge have been removed at hourly intervals. The position of the hole indicates that of the edge of the swarm when the sample was made. $\times 0.5$.

FIG. 2.-As fig. 1, swarm showing positions of hourly test samples, subsequently representing various aspects of the mature swarm, although in each case the advancing edge was actually sampled. $\times 1 \cdot 5$. 

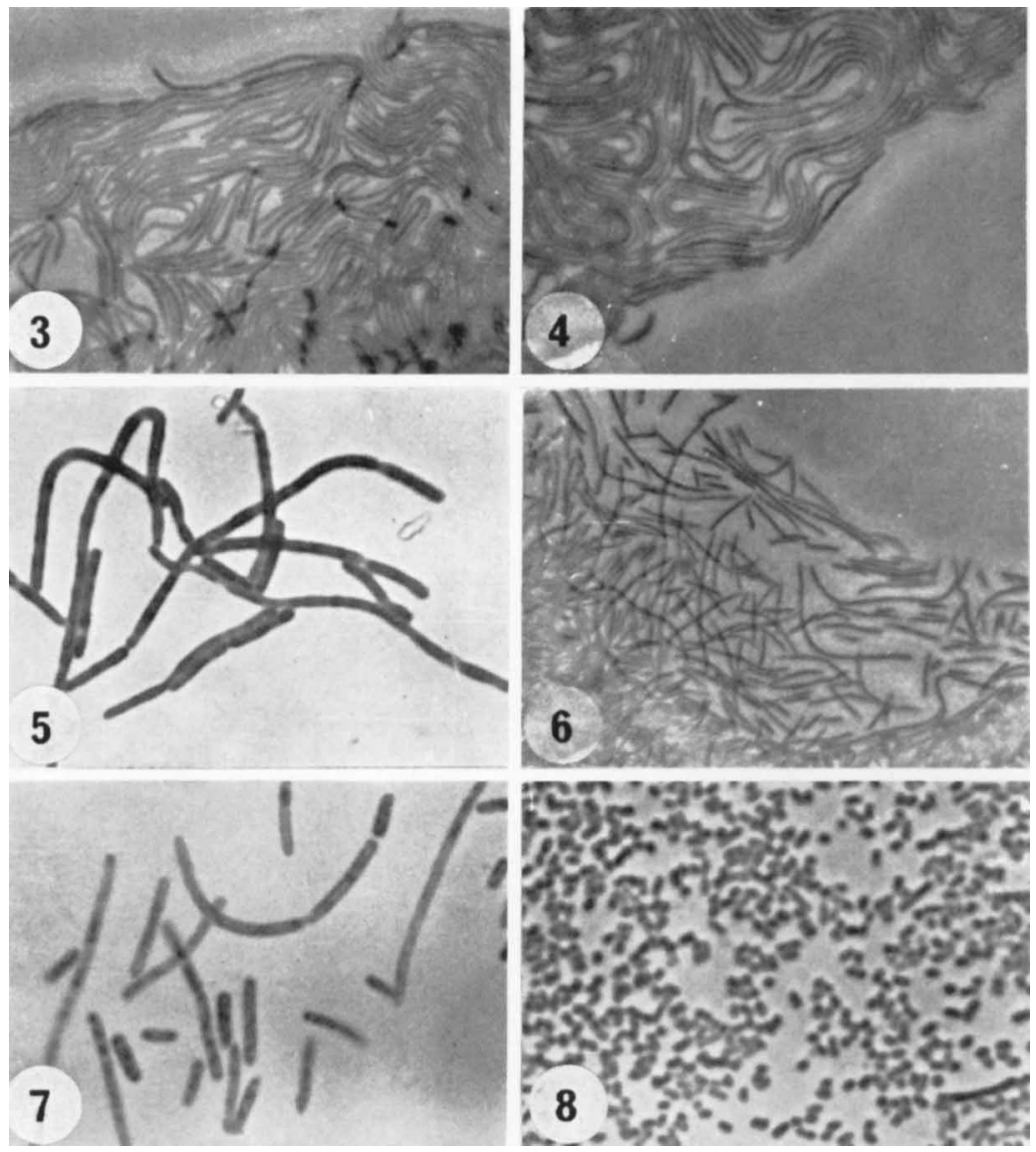

FIGS. 3 and 4.-The appearance of coiled filaments, composed of swarm elements, normally seen at the edge of the swarm, whether advancing or not; c. 5-10 h. Phase-contrast. $\times 1000$.

FIG. 5.-As figs. 3 and 4, dried smear from sample 3, stained with methylene blue, showing filamentous swarmers. $\times 1500$.

FIG. 6.-Exceptionally, the edge was disorganised but still composed of elongated swarm elements The appearance of the short elements, behind the edge, can be seen. Phase-contrast. $\times 1000$.

Fig. 7.-As fig. 6, dried smear from the same sample, stained with methylene blue, showing a mixture of filamentous swarmers and forms of medium length. $\times 1500$. This appearance is probably misleading, because the true non-swarmers are even smaller; compare with figs. 6 and 8 .

Fig. 8.-Dried smear stained with methylene blue, showing very short, non-swarming forms but including a single elongated swarmer; from the site of a previous edge sample near the centre of the growth, when complete, after a lapse of $12+\mathrm{h}$ and the cessation of swarming. $\times 1500$. Compare these forms with those in figs. 6 and 7, from a stationary edge of the swarm, during growth. 
Bisset, K. A. 1973b. The zonation phenomenon and structure of the swarm colony in Proteus mirabilis. J. med. Microbiol., 6, 429.

Wolstenholme, J. 1973. Evidence that the production of swollen bodies in the Dienes phenomenon may be confined to one of two interacting strains of Proteus mirabilis. J. gen. Microbiol., 74, 343. 